\title{
Stability of the Young and Hölder inequalities
}

\author{
Kazumasa Fujiwara ${ }^{1 *}$ and Tohru Ozawa ${ }^{2}$
}

${ }^{\text {"Correspondence: }}$

k-fujiwara@asagi.waseda.jp

${ }^{1}$ Department of Pure and Applied

Physics, Waseda University, Tokyo,

169-8555, Japan

Full list of author information is

available at the end of the article

\author{
Abstract \\ We give a simple proof of the Aldaz stability version of the Young and Hölder \\ inequalities and further refinements of available stability versions of those inequalities. \\ MSC: $26 \mathrm{D} 15$ \\ Keywords: Young inequality; Hölder inequality
}

\section{Introduction}

In this paper, we study the Young and Hölder inequalities from the point of view of the deviation from equalities with better upper and lower bound estimates. Particularly, we give a further refinement of Aldaz stability type inequalities [1] as well as a simple proof based exclusively on an algebraic argument with the standard Young inequality.

Throughout this paper, the following remainder function [2] plays an important role:

$$
R(\theta ; a, b)=\theta a+(1-\theta) b-a^{\theta} b^{1-\theta}
$$

where $a, b>0$ and $0 \leq \theta \leq 1$.

The standard Young inequality is described as

$$
R(\theta ; a, b) \geq 0
$$

which may be used without particular comments. The standard Hölder inequality follows from (1.2) and the equality

$$
\int_{\Omega}|f g| d \mu=\|f\|_{p}\|g\|_{p^{\prime}}\left(1-\int_{\Omega} R\left(\frac{1}{p} ; \frac{|f|^{p}}{\|f\|_{p}^{p}}, \frac{|g|^{p^{\prime}}}{\|g\|_{p^{\prime}}^{p^{\prime}}}\right) d \mu\right)
$$

for all $f \in L^{p}(\Omega, \mu) \backslash\{0\}$ and $g \in L^{p^{\prime}}(\Omega, \mu) \backslash\{0\}$, where $L^{q}(\Omega, \mu)$ is the Banach space of $q$ th integrable functions on a measure space $(\Omega, \mu)$ with the norm $\|\cdot\|_{q}, 1<q<\infty$, and $p^{\prime}$ is the dual exponent of $p$ defined by $1 / p+1 / p^{\prime}=1$.

The purpose in this paper is to give a clear understanding of the standard Young and Hölder inequalities on the basis of upper and lower bound estimates on the remainder function $R(\theta ; a, b)$. In Section 2, we reexamine the multiplication formula on $R(\theta ; a, b)[2]$ and present its dual formula. As a corollary, we give an algebraic proof of Aldaz stability type inequalities for the Young and Hölder inequalities [1]. In Section 3, we compare upper and lower bound estimates on $R(\theta ; a, b)$ in [1-4]. In Section 4 , we give dyadic refinements of the multiplication formulae on $R(\theta ; a, b)$ with their straightforward corollaries on (1.3) and discuss the associated dyadic refinements of the Hölder inequality.

@2014 Fujiwara and Ozawa; licensee Springer. This is an Open Access article distributed under the terms of the Creative Commons Attribution License (http://creativecommons.org/licenses/by/2.0), which permits unrestricted use, distribution, and reproduction in any medium, provided the original work is properly cited. 
There are many papers on the related subjects. We refer the reader to [1-7] and the references therein.

We close the introduction by giving some notation to be used in this paper. For $a, b \in \mathbb{R}$ we denote by $a \wedge b$ and $a \vee b$ their minimum and maximum, respectively.

\section{Multiplication formulae}

In this section, we revisit the original multiplication formula on $R(\theta ; a, b)[2]$ in connection with Aldaz stability type inequalities [1]. First of all, we recall Kichenassamy's multiplication formula.

Proposition 2.1 (Kichenassamy [2]) Let $\theta$ and $\sigma$ satisfy $0 \leq \theta, \sigma \leq 1$. Then the equality

$$
R(\theta \sigma ; a, b)=\theta R(\sigma ; a, b)+b^{1-\sigma} R\left(\theta ; a^{\sigma}, b^{\sigma}\right)
$$

holds for all $a, b>0$.

Proof The proposition follows from the equality

$$
\begin{aligned}
R(\sigma \theta, a, b) & =\sigma \theta a+(1-\theta \sigma) b-a^{\sigma \theta} b^{1-\sigma \theta} \\
& =\theta\left(\sigma a+(1-\sigma) b-a^{\sigma} b^{1-\sigma}\right)+\theta a^{\sigma} b^{1-\sigma}+(1-\theta) b-a^{\sigma \theta} b^{1-\sigma \theta} \\
& =\theta R(\sigma, a, b)+b^{1-\sigma}\left(\theta a^{\sigma}+(1-\theta) b^{\sigma}-a^{\sigma \theta} b^{\sigma(1-\theta)}\right) .
\end{aligned}
$$

Corollary 2.2 Let $\theta$ and $\sigma$ satisfy $0<\theta \leq \sigma<1$. Then the equality

$$
R(\theta ; a, b)=\frac{\theta}{\sigma} R(\sigma ; a, b)+b^{1-\sigma} R\left(\frac{\theta}{\sigma} ; a^{\sigma}, b^{\sigma}\right)
$$

holds for all $a, b>0$.

Proposition 2.3 Let $\theta$ and $\sigma$ satisfy $0 \leq \theta \leq \sigma<1$. Then the equality

$$
R(\sigma, a, b)=\frac{1-\sigma}{1-\theta} R(\theta, a, b)+a^{\theta} R\left(\frac{\sigma-\theta}{1-\theta}, a^{1-\theta}, b^{1-\theta}\right)
$$

holds for all $a, b>0$.

Remark 2.1 Equality (2.3) is regarded as a dual formula for $R(\theta ; a, b)$ in the sense that $\frac{1-\sigma}{1-\theta}+\frac{\sigma-\theta}{1-\theta}=1$.

Proof of Proposition 2.3

$$
\begin{aligned}
& R(\sigma, a, b) \\
&=\sigma a+(1-\sigma) b-a^{\sigma} b^{1-\sigma} \\
&=\frac{1-\sigma}{1-\theta}\left(\theta a+(1-\theta) b-a^{\theta} b^{1-\theta}\right)+\frac{\sigma-\theta}{1-\theta} a+\frac{1-\sigma}{1-\theta} a^{\theta} b^{1-\theta}-a^{\sigma} b^{1-\sigma} \\
&= \frac{1-\sigma}{1-\theta} R(\theta, a, b)+a^{\theta}\left(\frac{\sigma-\theta}{1-\theta} a^{1-\theta}+\frac{1-\sigma}{1-\theta} b^{1-\theta}-a^{\sigma-\theta} b^{(1-\theta)\left(1-\frac{\sigma-\theta}{1-\theta}\right)}\right) .
\end{aligned}
$$


Corollary 2.4 Let $\theta$ and $\sigma$ satisfy $0 \leq \theta \leq \sigma<1$. Then the equality

$$
R(\theta ; a, b)=\frac{1-\theta}{1-\sigma} R(\sigma ; a, b)-\frac{1-\theta}{1-\sigma} a^{\theta} R\left(\frac{\sigma-\theta}{1-\theta} ; a^{1-\theta}, b^{1-\theta}\right)
$$

holds for all $a, b>0$.

Remark 2.2 Propositions 2.1 and 2.3 are equivalent. In fact, it follows from the reciprocal formula $R(\theta ; a, b)=R(1-\theta, b, a)$ and Proposition 2.1 that

$$
\begin{aligned}
R(\sigma ; a, b) & =R(1-\sigma ; b, a) \\
& =\frac{1-\sigma}{1-\theta} R(1-\theta ; b, a)+a^{1-(1-\theta)} R\left(\frac{1-\sigma}{1-\theta} ; b^{1-\theta}, a^{1-\theta}\right) \\
& =\frac{1-\sigma}{1-\theta} R(\theta ; a, b)+a^{\theta} R\left(\frac{\sigma-\theta}{1-\theta} ; a^{1-\theta}, b^{1-\theta}\right),
\end{aligned}
$$

which is precisely (2.3). Conversely, given $\theta$ and $\sigma$ with $0<\theta \leq 1,0<\sigma \leq 1$, we put $\theta^{\prime}=$ $1-\theta \sigma$ and $\sigma^{\prime}=1-\sigma$. Then we have $0 \leq \sigma^{\prime} \leq \theta^{\prime}<1, \sigma=1-\sigma^{\prime}, \theta=\left(1-\theta^{\prime}\right) /\left(1-\sigma^{\prime}\right)$, and $\theta \sigma=1-\theta^{\prime}$. By the reciprocal formula and Proposition 2.3, we have

$$
\begin{aligned}
R(\theta \sigma ; a, b) & =R\left(1-\theta^{\prime} ; a, b\right)=R\left(\theta^{\prime} ; b, a\right) \\
& =\frac{1-\theta^{\prime}}{1-\sigma^{\prime}} R\left(\sigma^{\prime} ; b, a\right)+b^{\sigma^{\prime}} R\left(\frac{\theta^{\prime}-\sigma^{\prime}}{1-\sigma^{\prime}} ; b^{1-\sigma^{\prime}}, a^{1-\sigma^{\prime}}\right) \\
& =\frac{1-\theta^{\prime}}{1-\sigma^{\prime}} R\left(1-\sigma^{\prime} ; a, b\right)+b^{\sigma^{\prime}} R\left(\frac{1-\theta^{\prime}}{1-\sigma^{\prime}} ; a^{1-\sigma^{\prime}}, b^{1-\sigma^{\prime}}\right) \\
& =\theta R(\sigma ; a, b)+b^{1-\sigma} R\left(\theta ; a^{\sigma}, b^{\sigma}\right),
\end{aligned}
$$

which is precisely (2.1).

Proposition 2.5 (Aldaz [1], Kichenassamy [2]) Let $0 \leq \theta \leq 1$. Then the inequalities

$$
(\theta \wedge(1-\theta))\left(a^{1 / 2}-b^{1 / 2}\right)^{2} \leq R(\theta ; a, b) \leq(\theta \vee(1-\theta))\left(a^{1 / 2}-b^{1 / 2}\right)^{2}
$$

hold for all $a, b>0$.

Proof Though the first inequality of (2.5) is shown in [2], we show the inequalities in (2.5) for completeness. In the case $0 \leq \theta \leq 1 / 2$, we use Corollaries 2.2 and 2.4 with $\sigma=1 / 2$ to obtain

$$
\begin{aligned}
\theta\left(a^{1 / 2}-b^{1 / 2}\right)^{2} & =2 \theta R(1 / 2 ; a, b)=R(\theta ; a, b)-b^{1 / 2} R(2 \theta ; a, b) \\
& \leq R(\theta ; a, b) \\
& =2(1-\theta) R(1 / 2 ; a, b)-2(1-\theta) a^{\theta} R\left(\frac{1 / 2-\theta}{1-\theta} ; a^{1-\theta}, b^{1-\theta}\right) \\
& \leq 2(1-\theta) R(1 / 2 ; a, b)=(1-\theta)\left(a^{1 / 2}-b^{1 / 2}\right)^{2} .
\end{aligned}
$$


In the case $1 / 2 \leq \theta \leq 1$, we apply (2.6) with $\theta$ replaced by $1-\theta$ to obtain

$$
2(1-\theta) R(1 / 2 ; b, a) \leq R(1-\theta ; b, a) \leq 2 \theta R(1 / 2 ; b, a),
$$

which is precisely (2.5).

Remark 2.3 An equivalent couple of inequalities in Proposition 2.5 were proved by Aldaz [1] by differential calculus. The proof above depends on algebraic identities with the standard Young inequality.

\section{Upper and lower bounds of the remainder function}

In this section, we collect and compare several bounds of the remainder function $R(\theta ; a, b)$. For that purpose, we study the upper and lower bound estimates in terms of majorant $M(\theta ; a, b)$ and minorant $m(\theta ; a, b)$ in the form

$$
m(\theta ; a, b) \leq R(\theta ; a, b) \leq M(\theta ; a, b)
$$

for all $a, b>0$. We introduce four couples of the bounds as follows:

$$
\begin{aligned}
\text { [A] } \quad m_{A}(\theta ; a, b) & =(\theta \wedge(1-\theta))\left(a^{1 / 2}-b^{1 / 2}\right)^{2}, \\
M_{A}(\theta ; a, b) & =(\theta \vee(1-\theta))\left(a^{1 / 2}-b^{1 / 2}\right)^{2}, \\
{[\mathrm{~K}] \quad m_{K}(\theta ; a, b) } & =\frac{\theta(1-\theta)}{2}(a \wedge b)(\log a-\log b)^{2}, \\
M_{K}(\theta ; a, b) & =\frac{\theta(1-\theta)}{2}(a \vee b)(\log a-\log b)^{2}, \\
{[\mathrm{H}] \quad m_{H}(\theta ; a, b) } & =(\theta \wedge(1-\theta))\left|a^{\theta \wedge(1-\theta)}-b^{\theta \wedge(1-\theta)}\right|^{1 /(\theta \wedge(1-\theta))}, \\
M_{H}(\theta ; a, b) & =(\theta \vee(1-\theta))\left|a^{\theta \vee(1-\theta)}-b^{\theta \vee(1-\theta)}\right|^{1 /(\theta \vee(1-\theta))}, \\
{[\mathrm{FO}] \quad m_{F O}(\theta ; a, b) } & =\frac{\theta(1-\theta)}{2(a \vee b)}(a-b)^{2}, \\
M_{F O}(\theta ; a, b) & =\frac{\theta(1-\theta)}{2(a \wedge b)}(a-b)^{2} .
\end{aligned}
$$

Those couples are given respectively in $[1,2,4]$, and [3].

Remark 3.1 By the monotonicity property suggested in [2], the remainder function with respect to $\theta \in[0,1]$ is approximated arbitrarily precisely by the remainder functions with respect to rationals which approximate $\theta$. However, the approximation obtained by the monotonicity property is rather involved. Here, we focus only on lower and upper bounds with regard to a difference.

Simple relationships in those couples are summarized in the following.

Proposition 3.1 Let $0 \leq \theta \leq 1$. Then the inequalities

$$
m_{H}(\theta ; a, b) \leq m_{A}(\theta ; a, b) \leq R(\theta, a, b) \leq M_{A}(\theta ; a, b) \leq M_{H}(\theta, a, b),
$$




$$
m_{K}(\theta ; a, b) \leq m_{F O}(\theta ; a, b) \leq R(\theta, a, b) \leq M_{K}(\theta ; a, b) \leq M_{F O}(\theta, a, b)
$$

hold for all $a, b>0$.

Proof By homogeneity, (3.1) follows from the inequality

$$
\left(x^{\theta}-1\right)^{1 / \theta} \leq\left(x^{\sigma}-1\right)^{1 / \sigma}
$$

for all $x \geq 1$ and any $\theta$ and $\sigma$ with $0 \leq \theta \leq \sigma$. Inequality (3.3) follows from

$$
x^{\theta}=\left(x^{\sigma}-1+1\right)^{\theta / \sigma} \leq\left(x^{\sigma}-1\right)^{\theta / \sigma}+1
$$

Although some inequalities in (3.2) are proved in $[4,8]$, we prove (3.2) for completeness. By the integral representations $[4,8]$

$$
\begin{aligned}
R(\theta ; a, b) & =\theta(1-\theta)\left[\int_{0}^{1} \int_{0}^{t}(t a+(1-t) b)^{\theta-1}(s a+(1-s) b)^{-\theta} d s d t\right](a-b)^{2} \\
& =\left[\int_{0}^{1}((\theta(1-t)) \wedge((1-\theta) t)) a^{t} b^{1-t} d t\right](\log a-\log b)^{2}
\end{aligned}
$$

we have

$$
\begin{aligned}
& m_{F O}(\theta ; a, b) \leq R(\theta ; a, b) \leq M_{F O}(\theta ; a, b) \\
& m_{K}(\theta ; a, b) \leq R(\theta ; a, b) \leq M_{K}(\theta ; a, b) .
\end{aligned}
$$

Then it suffices to prove that

$$
\begin{aligned}
& m_{K}(\theta ; a, b) \leq m_{F O}(\theta ; a, b), \\
& M_{K}(\theta ; a, b) \leq M_{F O}(\theta ; a, b) .
\end{aligned}
$$

The last two inequalities are equivalent and follow from

$$
x(\log x)^{2} \leq(x-1)^{2}
$$

for all $x>0$.

Proposition 3.2 Let $0<\theta<1$ and let

$$
t_{0}(\theta)=\left(\sqrt{\frac{2}{(\theta \vee(1-\theta))}}-1\right)^{2} .
$$

Then the following inequalities hold for all $a, b>0$ :

$$
\begin{array}{ll}
m_{A}(\theta, a, b) \leq m_{F O}(\theta, a, b) & \text { if }(a \vee b) t_{0}(\theta) \leq a \wedge b, \\
m_{A}(\theta, a, b) \geq m_{F O}(\theta, a, b) & \text { if } 0<a \wedge b \leq(a \vee b) t_{0}(\theta) .
\end{array}
$$


Remark 3.2 Since $0<\theta \wedge(1-\theta) \leq 1 / 2 \leq \theta \vee(1-\theta)<1, t_{0}(\theta)$ satisfies

$$
(\sqrt{2}-1)^{2}<t_{0}(\theta) \leq 1
$$

for all $\theta$ with $0 \leq \theta \leq 1$. Proposition 3.2 shows that $m_{F O}(\theta ; a, b)$ is better than $m_{A}(\theta ; a, b)$ in a neighborhood of the diagonal $a=b$ in the quarter plane $(0, \infty) \times(0, \infty)$.

Proof of Proposition 3.2 It is sufficient to show inequalities (3.4) and (3.5) with $0<a<b$. We have

$$
\begin{aligned}
& \lim _{a \rightarrow 0} m_{A}(\theta, a, b)=(\theta \wedge(1-\theta)) b \geq \lim _{a \rightarrow 0} m_{F O}(\theta, a, b)=\frac{\theta(1-\theta)}{2} b, \\
& \lim _{a \rightarrow b} \frac{m_{A}(\theta, a, b)}{\left(a^{1 / 2}-b^{1 / 2}\right)^{2}}=\theta \wedge(1-\theta) \leq \lim _{a \rightarrow b} \frac{m_{F O}(\theta, a, b)}{\left(a^{1 / 2}-b^{1 / 2}\right)^{2}}=2 \theta(1-\theta) .
\end{aligned}
$$

Moreover, $m_{A}(\theta, a, b)=m_{F O}(\theta, a, b)$ is equivalent to the equation

$$
\left((a / b)^{1 / 2}+1\right)^{2}=\frac{2(\theta \wedge(1-\theta))}{\theta(1-\theta)} .
$$

Since the ratio of $a / b$ satisfying (3.6) with given $\theta$ is uniquely determined, inequalities (3.4) and (3.5) follow.

To compare $M_{A}$ and $M_{K}$, we prepare Lambert's $W$ function, which is defined as the inverse function of $[-1, \infty) \ni x \mapsto x e^{1 / x} \in[-1 / e, \infty)$. For details, see [8].

Proposition 3.3 Let $0 \leq \theta \leq 1$ and let

$$
t_{1}(\theta)=-\sqrt{2(\theta \wedge(1-\theta))} W\left(\frac{-1}{\sqrt{2(\theta \wedge(1-\theta))}} \exp \left(\frac{-1}{\sqrt{2(\theta \wedge(1-\theta))}}\right)\right),
$$

where $t_{1}(0)$ and $t_{1}(1)$ are understood to be

$$
\lim _{\theta \downarrow 0} t_{1}(\theta)=\lim _{\theta \uparrow 1} t_{1}(\theta)=1 .
$$

Then the following inequalities hold for any $a, b>0$ :

$$
\begin{array}{ll}
M_{A}(\theta ; a, b) \leq M_{K}(\theta ; a, b) & \text { if }(a \wedge b) \leq t_{1}(\theta)(a \vee b), \\
M_{A}(\theta ; a, b) \geq M_{K}(\theta ; a, b) & \text { if }(a \wedge b) \geq t_{1}(\theta)(a \vee b) .
\end{array}
$$

Remark 3.3 Since $0 \leq \theta \wedge(1-\theta) \leq 1 / 2, t_{1}(\theta)$ satisfies $0 \leq t_{1}(\theta) \leq 1$ for $0 \leq \theta \leq 1$. In the proof below, we see that $0<t_{1}(\theta)<1$ if $0<\theta<1$. Proposition 3.3 shows that $M_{K}(\theta ; a, b)$ is better than $M_{A}(\theta)$ in a neighborhood of the diagonal $a=b$ in the quarter plane $(0, \infty) \times$ $(0, \infty)$.

Proof of Proposition 3.3 Let $t>0$ satisfy $t^{2}=(a \wedge b) /(a \vee b)$. The magnitude correlation of $M_{A}(\theta, a, b)$ and $M_{K}(\theta ; a, b)$ coincides with that of

$$
\sqrt{(a \vee b)^{-1} M_{A}(\theta, a, b)}=1-t
$$


Table 1 The signs at the important values of $x$

\begin{tabular}{llllllll}
\hline $\boldsymbol{t}$ & $\mathbf{0}$ & $\cdots$ & $\boldsymbol{t}_{\mathbf{1}}(\boldsymbol{\theta})$ & $\cdots$ & $\sqrt{\mathbf{2 ( \boldsymbol { \theta } \wedge ( \boldsymbol { \theta } - \mathbf { 1 } ) )}}$ & $\cdots$ & $\mathbf{1}$ \\
\hline$f^{\prime}(t)$ & $\infty$ & + & + & + & 0 & - & - \\
$f(t)$ & $-\infty$ & $\nearrow$ & 0 & $\nearrow$ & + & $\searrow$ & 0 \\
\hline
\end{tabular}

and

$$
\sqrt{(a \vee b)^{-1} M_{A}(\theta, a, b)}=-\sqrt{2(\theta \wedge(1-\theta))} \log t .
$$

Let $f(t)=1-t+\sqrt{2(\theta \wedge(1-\theta))} \log (t)$. We have $f\left(t_{1}(\theta)\right)=0$ since

$$
\begin{aligned}
& \frac{-t_{1}(\theta)}{\sqrt{2(\theta \wedge(1-\theta))}} \exp \left(\frac{-t_{1}(\theta)}{\sqrt{2(\theta \wedge(1-\theta))}}\right) \\
& =\frac{-1}{\sqrt{2(\theta \wedge(1-\theta))}} \exp \left(\frac{-1}{\sqrt{2(\theta \wedge(1-\theta))}}\right),
\end{aligned}
$$

which is rewritten as

$$
\exp \left(\frac{1-t_{1}(\theta)}{\sqrt{2(\theta \wedge(1-\theta))}}\right)=t_{1}(\theta)^{-1}
$$

and, moreover,

$$
1-t_{1}(\theta)=-\sqrt{2(\theta \wedge(1-\theta))} \log \left(t_{1}(\theta)\right) .
$$

In addition,

$$
f^{\prime}(t)=-1+\sqrt{2(\theta \wedge(1-\theta))} / t
$$

Then inequalities (3.7) and (3.8) follow from the Table 1.

\section{Dyadic refinements of multiplication formulae and their applications}

In this section, we give dyadic refinements of the multiplication and dual multiplication formulae on the remainder function $R(\theta ; a, b)$ and their applications. By the reciprocal formula $R(\theta ; a, b)=R(1-\theta ; b, a)$, it is important to describe the formation of the remainder function as $\theta \rightarrow 0$ and $\theta \rightarrow 1 / 2$ with the principal terms $2 \theta R(1 / 2 ; a, b)$ and $2(1-\theta) R(1 / 2 ; a, b)$. For that purpose, we utilize dyadic decomposition.

Proposition 4.1 Let $\theta$ satisfy $0<\theta \leq 2^{-n}$ with an integer $n \geq 1$. Then the equality

$$
R(\theta, a, b)=\theta \sum_{j=1}^{n} 2^{j-1} b^{1-2^{1-j}}\left(a^{2^{-j}}-b^{2^{-j}}\right)^{2}+b^{1-2^{-n}} R\left(2^{n} \theta, a^{2^{-n}}, b^{2^{-n}}\right)
$$

holds for all $a, b>0$.

Proof We apply Corollary 2.2 with $\sigma=1 / 2$ to obtain

$$
R(\theta ; a, b)=\theta\left(a^{1 / 2}-b^{1 / 2}\right)+b^{1 / 2} R\left(2 \theta ; a^{1 / 2}, b^{1 / 2}\right),
$$




$$
\begin{array}{rl}
b^{1-2^{-j}} & R\left(2^{j} \theta ; a^{2^{-j}}, b^{2^{-j}}\right) \\
& =b^{1-2^{-j}}\left(2^{j+1} \theta R\left(1 / 2 ; a^{2^{-j}}, b^{2^{-j}}\right)+b^{2^{-j-1}} R\left(2^{j+1} \theta ; a^{2^{-j-1}}, b^{2^{-j-1}}\right)\right) \\
& =2^{j} \theta b^{1-2^{-j}}\left(a^{2^{-j}}-b^{2^{-j}}\right)^{2}+b^{1-2^{-j-1}} R\left(2^{j+1} \theta ; a^{2^{-j-1}}, b^{2^{-j-1}}\right)
\end{array}
$$

for any $j$ with $1 \leq j \leq n$. Then (4.1) follows immediately.

Proposition 4.2 Let $\theta$ satisfy $\left(2^{m-1}-1\right) /\left(2^{m}-1\right) \leq \theta \leq 1 / 2$ with an integer $m \geq 1$. Then the equality

$$
\begin{aligned}
R(\theta ; a, b)= & (1-\theta)\left(a^{1 / 2}-b^{1 / 2}\right)^{2} \\
& -(1-2 \theta) \sum_{j=1}^{m} 2^{j-1} a^{\theta} b^{(1-\theta)\left(1-2^{1-j}\right)}\left(a^{(1-\theta) 2^{-j}}-b^{(1-\theta) 2^{-j}}\right)^{2} \\
& -2(1-\theta) a^{\theta} b^{(1-\theta)\left(1-2^{-m}\right)} R\left(2^{m} \cdot \frac{1 / 2-\theta}{1-\theta} ; a^{(1-\theta) 2^{-m}}, b^{(1-\theta) 2^{-m}}\right)
\end{aligned}
$$

holds for all $a, b>0$.

Proof We apply Corollary 2.4 with $\sigma=1 / 2$ to obtain

$$
R(\theta ; a, b)=(1-\theta)\left(a^{1 / 2}-b^{1 / 2}\right)^{2}-2(1-2 \theta) a^{\theta} R\left(\frac{1 / 2-\theta}{1-\theta} ; a^{1-\theta}, b^{1-\theta}\right) .
$$

Then (4.2) follows by applying Proposition 4.1 to the last term on the right-hand side of (4.3) with $0 \leq(1 / 2-\theta) /(1-\theta) \leq 2^{-m}$.

Corollary 4.3 Let $0 \leq \theta \leq 1 / 2$. Then the inequalities

$$
\begin{aligned}
\theta( & \left(a^{1 / 2}-b^{1 / 2}\right)^{2}+(2 \theta \wedge(1-2 \theta)) b^{1 / 2}\left(a^{1 / 4}-b^{1 / 4}\right)^{2} \\
\leq & R(\theta ; a, b) \\
\leq & (1-\theta)\left(a^{1 / 2}-b^{1 / 2}\right)^{2}-(1-2 \theta) a^{\theta}\left(a^{(1-\theta) / 2}-b^{(1-\theta) / 2}\right)^{2} \\
& -2(\theta \wedge(1-2 \theta)) a^{\theta} b^{(1-\theta) / 2}\left(a^{(1-\theta) / 4}-b^{(1-\theta) / 4}\right)^{2}
\end{aligned}
$$

hold for all $a, b>0$.

Corollary 4.4 Let $1 / 2 \leq \theta \leq 1$. Then the inequalities

$$
\begin{aligned}
(1- & \theta)\left(a^{1 / 2}-b^{1 / 2}\right)^{2}+((2(1-\theta)) \wedge(2 \theta-1)) a^{1 / 2}\left(a^{1 / 4}-b^{1 / 4}\right)^{2} \\
\leq & R(\theta ; a, b) \\
\leq & \theta\left(a^{1 / 2}-b^{1 / 2}\right)^{2}-(2 \theta-1) b^{1-\theta}\left(a^{\theta / 2}-b^{\theta / 2}\right)^{2} \\
& -2((1-\theta) \wedge(2 \theta-1)) a^{(1-\theta) / 2} b^{\theta}\left(a^{\theta / 4}-b^{\theta / 4}\right)^{2}
\end{aligned}
$$

hold for all $a, b>0$. 
Remark 4.1 Some of the lower bounds in Corollaries 4.3 and 4.4 may be found already in [2], Section 3.2.

Remark 4.2 Inequalities (4.4) and (4.5) improve (2.5). Inequalities (2.5) become an equality when $\theta=1 / 2$, while (4.4) become an equality when $\theta=0,1 / 2$ and (4.5) become an equality when $\theta=1 / 2,1$.

We are now in a position to apply the equalities above to Hölder type inequalities.

Theorem 4.5 Let $p$ satisfy $2 \leq p<\infty$ and let $m$ and $n$ be unique integers satisfying

$$
\left\{\begin{array}{l}
2^{n} \leq p<2^{n+1}, \quad n \geq 1 \\
\left(2^{m+1}-1\right) /\left(2^{m}-1\right) \leq p<\left(2^{m}-1\right) /\left(2^{m-1}-1\right), \quad m \geq 1
\end{array}\right.
$$

Then the equalities

$$
\begin{aligned}
& \|f\|_{p}\|g\|_{p^{\prime}}\left(1-\frac{1}{p^{\prime}} \int_{\Omega}\left(\frac{|f|^{p / 2}}{\|f\|_{p}^{p / 2}}-\frac{|g|^{p^{\prime} / 2}}{\|g\|_{p^{\prime}}^{p^{\prime} / 2}}\right)^{2} d \mu\right. \\
& +\left(\frac{1}{p^{\prime}}-\frac{1}{p}\right) \sum_{j=1}^{m} 2^{j-1} \int_{\Omega} \frac{|f|}{\|f\|_{p}} \frac{|g|^{1-2^{1-j}}}{\|g\|_{p^{\prime}}^{1-2^{1-j}}}\left(\frac{|f|^{(p-1) 2^{-j}}}{\|f\|_{p}^{(p-1) 2^{-j}}}-\frac{|g|^{2^{-j}}}{\|g\|_{p^{\prime}}^{2^{-j}}}\right)^{2} d \mu \\
& \left.\quad+\frac{2}{p^{\prime}} \int_{\Omega} \frac{|f|}{\|f\|_{p}} \frac{|g|^{\left(1-2^{-m}\right)}}{\|g\|_{p^{\prime}}^{\left(1-2^{-m}\right)}} R\left(2^{m-1} \frac{p-2}{p-1}, \frac{|f|^{(p-1) 2^{-m}}}{\|f\|_{p}^{(p-1) 2^{-m}}}, \frac{|g|^{2^{-m}}}{\|g\|_{p^{\prime}}^{2^{-m}}}\right) d \mu\right) \\
& =\|f g\|_{1} \\
& =\|f\|_{p}\|g\|_{p^{\prime}}\left(1-\frac{1}{p} \sum_{j=1}^{n} 2^{j-1} \int_{\Omega} \frac{|g|^{p^{\prime}\left(1-2^{1-j}\right)}}{\|g\|_{p^{\prime}}^{p^{\prime}\left(1-2^{1-j}\right)}}\left(\frac{|f|^{p^{-j}}}{\|f\|_{p}^{p 2^{-j}}}-\frac{|g|^{p^{2^{2}-j}}}{\|g\|_{p^{\prime}}^{p^{\prime} 2^{-j}}}\right)^{2} d \mu\right. \\
& \left.\quad-\int_{\Omega} \frac{|g|^{p^{\prime}\left(1-2^{-n}\right)}}{\|g\|_{p^{\prime}}^{p^{\prime}\left(1-2^{-n}\right)}} R\left(\frac{2^{n}}{p}, \frac{|f|^{p^{2}-n}}{\|f\|_{p}^{p 2^{-n}}}, \frac{|g|^{p^{\prime} 2^{-n}}}{\|g\|_{p^{\prime}}^{p^{\prime} 2^{-n}}}\right) d \mu\right)
\end{aligned}
$$

hold for all $f \in L^{p}(\Omega, \mu) \backslash\{0\}$ and $g \in L^{p^{\prime}}(\Omega, \mu) \backslash\{0\}$.

Proof The theorem follows from (1.3) and Propositions 4.1 and 4.2 with $\theta=1 / p, a=$ $|f|^{p} /\|f\|_{p}^{p}, b=|g|^{p^{\prime}} /\|g\|_{p^{\prime}}^{p^{\prime}}$.

Corollary 4.6 Let $p, m, n$ be as in Theorem 4.5. Then the inequalities

$$
\begin{aligned}
\|f\|_{p}\|g\|_{p^{\prime}}\left(1-\frac{1}{p^{\prime}} \int_{\Omega}\left(\frac{|f|^{p / 2}}{\|f\|_{p}^{p / 2}}-\frac{|g|^{p^{\prime} / 2}}{\|g\|_{p^{\prime}}^{p^{\prime} / 2}}\right)^{2} d \mu\right. \\
+\left(\frac{1}{p^{\prime}}-\frac{1}{p}\right) \sum_{j=1}^{m} 2^{j-1} \int_{\Omega} \frac{|f|}{\|f\|_{p}} \frac{|g|^{1-2^{1-j}}}{\|g\|_{p^{\prime}}^{12^{1-j}}}\left(\frac{|f|^{(p-1) 2^{-j}}}{\|f\|_{p}^{(p-1) 2^{-j}}}-\frac{|g|^{2^{-j}}}{\|g\|_{p^{\prime}}^{2^{-j}}}\right)^{2} d \mu \\
\left.+\frac{2}{p^{\prime}}\left(1-2^{m-1} \frac{p-2}{p-1}\right) \int_{\Omega} \frac{|f|}{\|f\|_{p}} \frac{|g|^{\left(1-2^{-m}\right)}}{\|g\|_{p^{\prime}}^{\left(1-2^{-m}\right)}}\left(\frac{|f|^{(p-1) 2^{-m-1}}}{\|f\|_{p}^{(p-1) 2^{-m-1}}}-\frac{|g|^{2^{-m-1}}}{\|g\|_{p^{\prime}}^{2^{-m-1}}}\right)^{2} d \mu\right)
\end{aligned}
$$


$\leq\|f g\|_{1}$

$$
\begin{aligned}
& \leq\|f\|_{p}\|g\|_{p^{\prime}}\left(1-\frac{1}{p} \sum_{j=1}^{n} 2^{j-1} \int_{\Omega} \frac{|g|^{p^{\prime}\left(1-2^{1-j}\right)}}{\|g\|_{p^{\prime}}^{p^{\prime}\left(1-2^{1-j}\right)}}\left(\frac{|f|^{p^{2}-j}}{\|f\|_{p}^{p^{-j}}}-\frac{|g|^{p^{\prime} 2^{-j}}}{\|g\|_{p^{\prime}}^{p^{\prime} 2^{-j}}}\right)^{2} d \mu\right. \\
& \left.-\frac{p-2^{n}}{p} \int_{\Omega} \frac{|g|^{p^{\prime}\left(1-2^{-n}\right)}}{\|g\|_{p^{\prime}}^{p^{\prime}\left(1-2^{-n}\right)}}\left(\frac{|f|^{p^{2-n-1}}}{\|f\|_{p}^{p 2^{-n-1}}}-\frac{|g|^{p^{\prime} 2^{-n-1}}}{\|g\|_{p^{\prime}}^{p^{\prime} 2^{-n-1}}}\right)^{2} d \mu\right)
\end{aligned}
$$

hold for all $f \in L^{p}(\Omega, \mu) \backslash\{0\}$ and $g \in L^{p^{\prime}}(\Omega, \mu) \backslash\{0\}$.

Proof The required inequalities follow from Theorem 4.5 and Proposition 2.5.

Corollary 4.7 Let $p \geq 2^{n}$ with a positive integer $n$. Then the inequalities

$$
\begin{aligned}
& \|f\|_{p}\|g\|_{p^{\prime}}\left(1-\frac{1}{p^{\prime}}\left\|\frac{|f|^{p / 2}}{\|f\|_{p}^{p / 2}}-\frac{|g|^{p^{\prime} / 2}}{\|g\|_{p^{\prime}}^{p^{\prime} / 2}}\right\|_{2}^{2}\right. \\
& \left.+\left(\frac{1}{p^{\prime}}-\frac{1}{p}\right)\left\|\frac{|f|^{1 / 2}}{\|f\|_{p}^{1 / 2}}\left(\frac{|f|^{(p-1) / 2}}{\|f\|_{p}^{(p-1) / 2}}-\frac{|g|^{1 / 2}}{\|g\|_{p^{\prime}}^{1 / 2}}\right)\right\|_{2}^{2}\right) \\
& \leq\|f g\|_{1} \\
& \leq\|f\|_{p}\|g\|_{p^{\prime}}\left(1-\frac{1}{p} \sum_{j=1}^{n} 2^{j-1}\left\|\frac{|g|^{p^{\prime}\left(1 / 2-2^{-j}\right)}}{\|g\|_{p^{\prime}}^{p^{\prime}\left(1 / 2-2^{-j}\right)}}\left(\frac{|f|^{p^{-j}}}{\|f\|_{p}^{p 2^{-j}}}-\frac{|g|^{p^{\prime} 2^{-j}}}{\|g\|_{p^{\prime}}^{p^{\prime} 2^{-j}}}\right)\right\|_{2}^{2}\right. \\
& \left.\quad-\frac{p-2^{n}}{p}\left\|\frac{|g|^{p^{\prime}\left(1 / 2-2^{-n-1}\right)}}{\|g\|_{p^{\prime}}^{p^{\prime}\left(1 / 2-2^{-n-1)}\right.}}\left(\frac{|f|^{p^{-n-1}}}{\|f\|_{p}^{p 2^{-n-1}}}-\frac{|g|^{p^{\prime} 2^{-n-1}}}{\|g\|_{p^{\prime}}^{p^{\prime} 2^{-n-1}}}\right)\right\|_{2}^{2}\right)
\end{aligned}
$$

hold for all $f \in L^{p}(\Omega, \mu) \backslash\{0\}$ and $g \in L^{p^{\prime}}(\Omega, \mu) \backslash\{0\}$.

Remark 4.3 In the case where $n=1$ in Corollary 4.7, the coefficients of the upper and lower bounds of $\|f g\|_{1}$ are symmetric as follows:

$$
\begin{aligned}
& \|f\|_{p}\|g\|_{p^{\prime}}\left(1-\frac{1}{p^{\prime}}\left\|\frac{|f|^{p / 2}}{\|f\|_{p}^{p / 2}}-\frac{|g|^{p^{\prime} / 2}}{\|g\|_{p^{\prime}}^{p^{\prime} / 2}}\right\|_{2}^{2}\right. \\
& \left.+\left(\frac{1}{p^{\prime}}-\frac{1}{p}\right)\left\|\frac{|f|^{1 / 2}}{\|f\|_{p}^{1 / 2}}\left(\frac{|f|^{(p-1) / 2}}{\|f\|_{p}^{(p-1) / 2}}-\frac{|g|^{1 / 2}}{\|g\|_{p^{\prime}}^{1 / 2}}\right)\right\|_{2}^{2}\right) \\
& \leq\|f g\|_{1} \\
& \leq\|f\|_{p}\|g\|_{p^{\prime}}\left(1-\frac{1}{p}\left\|\frac{|f|^{p / 2}}{\|f\|_{p}^{p / 2}}-\frac{|g|^{p^{\prime} / 2}}{\|g\|_{p^{\prime}}^{p^{\prime} / 2}}\right\|_{2}^{2}\right. \\
& \left.\quad-\left(\frac{1}{p^{\prime}}-\frac{1}{p}\right)\left\|\frac{|g|^{p^{\prime} / 4}}{\|g\|_{p^{\prime}}^{p^{\prime} / 4}}\left(\frac{|f|^{p / 4}}{\|f\|_{p}^{p / 4}}-\frac{|g|^{p^{\prime} / 4}}{\|g\|_{p^{\prime}}^{p^{\prime} / 4}}\right)\right\|_{2}^{2}\right) .
\end{aligned}
$$


Remark 4.4 Inequalities (4.8) improve the Aldaz stability version of the Hölder inequality [1]

$$
\begin{aligned}
& \|f\|_{p}\|g\|_{p^{\prime}}\left(1-\frac{1}{p^{\prime}}\left\|\frac{|f|^{p / 2}}{\|f\|_{p}^{p / 2}}-\frac{|g|^{p^{\prime} / 2}}{\|g\|_{p^{\prime}}^{p^{p^{\prime}}}}\right\|_{2}^{2}\right) \\
& \leq\|f g\|_{1} \leq\|f\|_{p}\|g\|_{p^{\prime}}\left(1-\frac{1}{p}\left\|\frac{|f|^{p / 2}}{\|f\|_{p}^{p / 2}}-\frac{|g|^{p^{\prime} / 2}}{\|g\|_{p^{\prime}}^{p^{\prime} / 2}}\right\|_{2}^{2}\right) .
\end{aligned}
$$

As Aldaz observed, (4.9) become

$$
\|f\|_{p}\|g\|_{p^{\prime}}\left(1-\frac{2}{p^{\prime}}\right) \leq\|f g\|_{1}=0 \leq\|f\|_{p}\|g\|_{p^{\prime}}\left(1-\frac{2}{p}\right)
$$

if $\operatorname{supp} f \cap \operatorname{supp} g=\emptyset$. In this respect, Corollary 4.7 is sharp since both sides of the inequalities in (4.8) vanish as follows:

$$
\begin{aligned}
& \|f\|_{p}\|g\|_{p^{\prime}}\left(1-\frac{1}{p^{\prime}}\left\|\frac{|f|^{p / 2}}{\|f\|_{p}^{p / 2}}-\frac{|g|^{p^{\prime} / 2}}{\|g\|_{p^{\prime}}^{p^{\prime} / 2}}\right\|_{2}^{2}\right. \\
& \left.+\left(\frac{1}{p^{\prime}}-\frac{1}{p}\right)\left\|\frac{|f|^{1 / 2}}{\|f\|_{p}^{1 / 2}}\left(\frac{|f|^{(p-1) / 2}}{\|f\|_{p}^{(p-1) / 2}}-\frac{|g|^{1 / 2}}{\|g\|_{p^{\prime}}^{1 / 2}}\right)\right\|_{2}^{2}\right) \\
& =\|f\|_{p}\|g\|_{p^{\prime}}\left(1-\frac{2}{p^{\prime}}+\frac{1}{p^{\prime}}-\frac{1}{p}\right)=0, \\
& \|f\|_{p}\|g\|_{p^{\prime}}\left(1-\frac{1}{p} \sum_{j=1}^{n} 2^{j-1}\left\|\frac{|g|^{p^{\prime}\left(1 / 2-2^{-j}\right)}}{\|g\|_{p^{\prime}}^{p^{\prime}\left(1 / 2-2^{-j}\right)}}\left(\frac{|f|^{p^{2-j}}}{\|f\|_{p}^{p^{2-j}}}-\frac{|g|^{p^{\prime} 2^{-j}}}{\|g\|_{p^{\prime}}^{p^{p^{2}-j}}}\right)\right\|_{2}^{2}\right. \\
& \left.-\frac{p-2^{n}}{p}\left\|\frac{|g|^{p^{\prime}\left(1 / 2-2^{-n-1}\right)}}{\|g\|_{p^{\prime}}^{p^{\prime}\left(1 / 2-2^{-n-1}\right)}}\left(\frac{|f|^{p^{-n-1}}}{\|f\|_{p}^{p 2^{-n-1}}}-\frac{|g|^{p^{\prime} 2^{-n-1}}}{\|g\|_{p^{\prime}}^{p^{\prime} 2^{-n-1}}}\right)\right\|_{2}^{2}\right) \\
& =\|f\|_{p}\|g\|_{p^{\prime}}\left(1-\frac{2}{p}-\frac{1}{p} \sum_{j=2}^{n} 2^{j-1}-\frac{p-2^{n}}{p}\right)=0 .
\end{aligned}
$$

In addition, (4.8) coincides with the polarization identity

$$
(|f|,|g|)=\|f\|_{2}\|g\|_{2}\left(1-\frac{1}{2}\left\|\frac{|f|}{\|f\|_{2}}-\frac{|g|}{\|g\|_{2}}\right\|_{2}^{2}\right)
$$

when $p=2$, where $(\cdot, \cdot)$ is the standard $L^{2}$ inner product. 


\section{Acknowledgements}

The authors are grateful to the referees for important remarks and suggestions.

Received: 18 January 2014 Accepted: 11 April 2014 Published: 06 May 2014

\section{References}

1. Aldaz, JM: A stability version of Hölder's inequality. J. Math. Anal. Appl. 343, 842-852 (2008)

2. Kichenassamy, S: Improving Hölder's inequality. Houst. J. Math. 36, 303-312 (2010)

3. Fujiwara, K, Ozawa, T: Exact remainder formula for the Young inequality and applications. Int. J. Math. Anal. 7, 2733-2735 (2013)

4. Hu, X-L: An extension of Young's inequality and its application. Appl. Math. Comput. 219, 6393-6399 (2013)

5. Furuichi, S, Minculete, N: Alternative reverse inequalities for Young's inequality. J. Math. Inequal. 5, 595-600 (2011)

6. Gao, X, Gao, M, Shang, X: A refinement of Hölder's inequality and applications. JIPAM. J. Inequal. Pure Appl. Math. 8, Article ID 44 (2007)

7. Pečarić, J, Šimić, V: A note on the Hölder inequality. JIPAM. J. Inequal. Pure Appl. Math. 7, Article ID 176 (2006)

8. Corless, RM, Gonnet, GH, Hare, DEG, Jeffrey, DJ, Knuth, DE: On the Lambert W function. Adv. Comput. Math. 5, 329-359 (1996)

10.1186/1029-242X-2014-162

Cite this article as: Fujiwara and Ozawa: Stability of the Young and Hölder inequalities. Journal of Inequalities and Applications 2014, 2014:162

\section{Submit your manuscript to a SpringerOpen ${ }^{\circ}$ journal and benefit from:}

- Convenient online submission

- Rigorous peer review

- Immediate publication on acceptance

- Open access: articles freely available online

- High visibility within the field

- Retaining the copyright to your article 